Discrete Comput Geom 37:301-320 (2007)

DOI: $10.1007 /$ s00454-006-1299-y

\title{
Volume and Lattice Points of Reflexive Simplices
}

\author{
Benjamin Nill \\ Research Group Lattice Polytopes, FU Berlin, \\ Arnimallee 3, 14195 Berlin, Germany \\ nill@math.fu-berlin.de
}

Communicated by Günter M. Ziegler

\begin{abstract}
Using new number-theoretic bounds on the denominators of unit fractions summing up to one, we show that in any dimension $d \geq 4$ there is only one $d$-dimensional reflexive simplex having maximal volume. Moreover, only these reflexive simplices can admit an edge that has the maximal number of lattice points possible for an edge of a reflexive simplex. In general, these simplices are also expected to contain the largest number of lattice points even among all lattice polytopes with only one interior lattice point. Translated in algebro-geometric language, our main theorem yields a sharp upper bound on the anticanonical degree of $d$-dimensional $\mathbb{Q}$-factorial Gorenstein toric Fano varieties with Picard number one, e.g., of weighted projective spaces with Gorenstein singularities.
\end{abstract}

\section{Introduction}

To make this article as accessible as possible to readers from different backgrounds, we summarize our results from the points of view of convex geometry, algebraic geometry, and number theory, respectively.

Convex and Discrete Geometry. Given a $d$-dimensional lattice polytope $P$ with only one lattice point in its interior, Hensley [13] showed that the volume and the number of lattice points of $P$ is bounded above by a function depending only on $d$. In [21] and [25] asymptotically better upper bounds were obtained. However, in lower dimensions they are presumably still very far from being sharp, see [17] and [27]. Nevertheless, in any dimension $d \geq 3$ there exists an explicit candidate that is expected to yield the maximal values. This is a simplex, here called $S_{Q_{d}^{\prime}}$, that was already described more than 20 years ago [29]: $S_{Q_{d}^{\prime}}$ is (up to translation) the convex hull of the origin of the lattice and $y_{0} e_{0}, \ldots, y_{d-2} e_{d-2}, 2\left(y_{d-1}-1\right) e_{d-1}$, where $e_{0}, \ldots, e_{d-1}$ is a lattice basis and $\left(y_{0}, y_{1}, y_{2}, y_{3}, \ldots\right)=(2,3,7,43, \ldots)$ is the so-called Sylvester sequence. 
In [2] Batyrev introduced reflexive polytopes, which are lattice polytopes $P$, having the origin of the lattice in its interior, whose dual polytopes $P^{*}$ are also lattice polytopes. From the convex-geometric point of view, reflexive polytopes are special lattice polytopes with only one interior lattice point, and, since there are already in dimension four nearly half a billion isomorphism classes [18], they can be seen as a good testing ground for more general conjectures.

Recently, Haase and Melnikov noticed [12] that $S_{Q_{d}^{\prime}}$ is actually a reflexive simplex. Now, in the present paper we prove that in dimension $d \geq 4$ the simplices $S_{Q_{d}^{\prime}}$ are the only reflexive simplices having maximal volume (Theorem A). This also yields an upper bound on the number of lattice points of reflexive simplices (Corollary 1.6). Moreover, we show that there is a sharp bound on the number of lattice points of an edge of a reflexive simplex, which is only obtained by $S_{Q_{d}^{\prime}}$ (Theorem B). This was first observed by Haase and Melnikov in [12] for $d \leq 4$. Finally, we give a sharp upper bound on the product of the volumes of dual reflexive simplices (Theorem C). This can be seen as an isoperimetric inequality of Blaschke-Santaló type for reflexive simplices.

Algebraic and Toric Geometry. The Borisov-Alexeev conjecture states that $d$-dimensional Fano varieties with $\varepsilon$-log terminal singularities form a bounded family, in particular their anticanonical degree is bounded. This conjecture is of importance in birational geometry, and was proven in the toric case in [4]. However, good upper bounds are known only in particular cases. For instance, it was conjectured that $(d+1)^{d}$ is the maximal degree of a nonsingular Fano variety with Picard number one, achieved only for $\mathbb{P}^{d}$. This was proven for $d \leq 4$ in [15], however, it is wrong without the assumption of nonsingularity. For higher Picard numbers this bound does not hold anymore, see [1]. In Theorem 9 of [7] Debarre showed as a good approximation $\left(-K_{X}\right)^{d} \leq d ! d^{\rho d}$ for a $d$-dimensional nonsingular toric Fano variety $X$ with Picard number $\rho \geq 2$.

Now, in [26] an effective bound (namely 72) was given for canonical Gorenstein Fano threefolds, proving the so-called Fano-Iskovskikh conjecture. Proposing the conjectural value in higher dimensions, we provide here in Theorem $\mathrm{A}^{\prime}$ a sharp upper bound (namely $\left.2\left(y_{d-1}-1\right)^{2}\right)$ on the anticanonical degree $\left(-K_{X}\right)^{d}$ of (necessarily canonical) Gorenstein toric Fano varieties $X$ of dimension $d \geq 4$, under the additional assumptions that $X$ is $\mathbb{Q}$-factorial with Picard number one. Moreover, we have an effective bound on the anticanonical degree $-K_{X}$. $C$ of torus-invariant integral curves $C$ on such $X$ (Theorem $\left.\mathrm{B}^{\prime}\right)$. In both theorems we can completely characterize the cases of equality.

These results are based on the correspondence of Gorenstein toric Fano varieties via toric geometry to reflexive polytopes. These objects have been thoroughly studied, since the natural duality of reflexive polytopes yields a construction of mirror-symmetric Calabi-Yau varieties as general anticanonical hypersurfaces of the associated Gorenstein toric Fano varieties, see [2].

Stimulating further research we pose here the following question: While Theorem $\mathrm{A}^{\prime}$ determines in any dimension the maximal anticanonical degree of a weighted projective space with Gorenstein singularities, we would like to know what is the minimal anticanonical degree a $d$-dimensional weighted projective space with Gorenstein singularities can have? Could it be $(d+1)$ !? 
Elementary Number Theory. It is well known that questions concerning the volume and lattice points of simplices are closely related to the geometry of numbers and diophantine equations. Here, the crucial objects of investigation are finite families of positive natural numbers whose reciprocals sum up to one, e.g., $(2,3,6)$, since $\frac{1}{2}+\frac{1}{3}+\frac{1}{6}=1$. We call these unit partitions. In the study of these objects the Sylvester sequence plays a crucial role, as exemplified by a famous result of Curtiss [6]. Now, the proof of above results is based on new inequalities on unit partitions (Theorem 5.1). They were achieved by a method of proof first used by Izboldin and Kurliandchik in [16].

Organization of the Paper. This paper is organized in the following way: In the first section we start with the basic definitions and the main convex-geometric results (Theorems $\mathrm{A}, \mathrm{B}$, and $\mathrm{C}$ ). In the second section we give their algebro-geometric formulation (Theorems $\mathrm{A}^{\prime}, \mathrm{B}^{\prime}$, and $\mathrm{C}^{\prime}$ ). The third section contains a discussion of the correspondence between weighted projective spaces, weight systems, and lattice simplices containing the origin in their interiors. The fourth section then relates reflexive simplices and unit partitions, i.e., unit fractions summing up to one. In the fifth section we present our main number-theoretic result (Theorem 5.1). Finally, the last section contains the proofs of Theorems A, B, and C.

Remark. One should note that no knowledge of algebraic or toric geometry is necessary to understand the convex-geometric results and their proofs. Moreover, basic facts from toric geometry that are freely used in this article like the dictionary of fans/lattice polytopes and toric varieties can be found in [9]. All relevant properties of reflexive polytopes like their correspondence to Gorenstein toric Fano varieties are contained in [2] or [24].

\section{The Main Results, Convex-Geometric}

Throughout the paper let $d \geq 2$.

Let $N, M$ be dual lattices of rank $d$, with the pairing $\langle\cdot, \cdot\rangle: M \times N \rightarrow \mathbb{Z}$. We define $N_{\mathbb{R}}:=N \otimes_{\mathbb{Z}} \mathbb{R}$ and $M_{\mathbb{R}}:=M \otimes_{\mathbb{Z}} \mathbb{R}$. A lattice polytope (in $M_{\mathbb{R}}$ ) is a polytope whose vertices are lattice points (in $M$ ). Two lattice polytopes $P_{1}$ and $P_{2}$ are regarded as isomorphic or unimodularly equivalent, if there is a lattice isomorphism inducing an affine bijection between the affine hulls of $P_{1}$ and $P_{2}$ which maps every $i$-dimensional face of $P_{1}$ onto an $i$-dimensional face of $P_{2}$, for all $i, 0 \leq i \leq \operatorname{dim}\left(P_{1}\right)=\operatorname{dim}\left(P_{2}\right)$. In this case we write $P_{1} \cong P_{2}$. Moreover, if $P \subseteq M_{\mathbb{R}}$ is a lattice polytope, then $\operatorname{Vol}(P)$ denotes the (normalized) volume of $P$, i.e., $d$ ! times the euclidean volume of $P$ with respect to a lattice basis of $M$.

In [2] Batyrev introduced the following notion:

Definition 1.1. A reflexive polytope $P \subseteq M_{\mathbb{R}}$ is a $d$-dimensional lattice polytope containing the origin in its interior whose dual polytope $P^{*}$ is also a lattice polytope, where 
the dual polytope is defined as

$$
P^{*}:=\left\{x \in N_{\mathbb{R}}:\langle y, x\rangle \geq-1, \forall y \in P\right\} .
$$

Hence, duals of reflexive polytopes are again reflexive.

Now we need an elementary number-theoretic notion in order to construct some special reflexive simplices:

Definition 1.2. The well-known recursive sequence [28, A000058] of pairwise coprime natural numbers $y_{0}:=2, y_{n}:=1+y_{0} \cdots y_{n-1}(n \geq 1)$ is called the Sylvester sequence. It satisfies $y_{n}=y_{n-1}^{2}-y_{n-1}+1$ and starts as $y_{0}=2, y_{1}=3, y_{2}=7, y_{3}=43$, $y_{4}=1807$. We also define $t_{n}:=y_{n}-1$ for $n \in \mathbb{N}_{\geq 0}$.

Using these numbers we define two special families:

\section{Definition 1.3.}

- The $(d+1)$-tuple of natural numbers

$$
Q_{d}:=\left(\frac{t_{d}}{y_{0}}, \ldots, \frac{t_{d}}{y_{d-1}}, 1\right)
$$

is called the Sylvester weight system of length $d$.

- The $(d+1)$-tuple of natural numbers

$$
Q_{d}^{\prime}:=\left(\frac{2 t_{d-1}}{y_{0}}, \ldots, \frac{2 t_{d-1}}{y_{d-2}}, 1,1\right)
$$

is called the enlarged Sylvester weight system of length $d$.

We can define lattice simplices from these weight systems:

Definition 1.4. Let $Q$ be a $(d+1)$-tuple $\left(q_{0}, \ldots, q_{d}\right)$ of positive natural numbers such that $q_{d}=1$. Let $|Q|:=q_{0}+\cdots+q_{d}$. If $k_{i}:=|Q| / q_{i}$ is a natural number for any $i=0, \ldots, d-1$, then we define

$$
S_{Q}:=\operatorname{conv}\left(k_{0} e_{0}-u, \ldots, k_{d-1} e_{d-1}-u,-u\right),
$$

for $u:=e_{0}+\cdots+e_{d-1}$, where $e_{0}, \ldots, e_{d-1}$ is a $\mathbb{Z}$-basis of $M$.

For such $Q$ (in particular for $Q_{d}$ and $Q_{d}^{\prime}$ ) the associated simplices $S_{Q}$ are reflexive, see Proposition 4.4. Now, here is our first main result:

\section{Theorem A.}

1. $S_{(1,1,1)}$ is the unique two-dimensional reflexive simplex with the largest volume 9 , respectively the largest number of lattice points 10 . 
2. $S_{(3,1,1,1)}$ and $S_{Q_{3}^{\prime}}$ are the only three-dimensional reflexive simplices with the largest volume 72, respectively the largest number of lattice points 39.

3. Let $d \geq 4$. Then $S_{Q_{d}^{\prime}}$ is the unique d-dimensional reflexive simplex with the largest volume, namely $2 t_{d-1}^{2}$.

This bound vastly improves on general upper bounds on the volume of lattice simplices containing only one lattice point in the interior, as given in [21] or [25].

From the following theorem in [3] we also get an upper bound on the number of lattice points:

Lemma 1.5 (Blichfeldt). Let $P \subseteq M_{\mathbb{R}}$ be a d-dimensional lattice polytope. Then $|P \cap M| \leq d+\operatorname{Vol}(P)$.

Now, using a lower bound on the number of lattice points in $S_{Q_{d}^{\prime}}$ due to Zaks et al. [29] we see that there is still a gap to bridge:

Corollary 1.6. Let J denote the maximal number of lattice points some d-dimensional reflexive simplex can have. Then we get for $d \geq 3$,

$$
\frac{1}{3(d-2) !} t_{d-1}^{2}<J \leq d+2 t_{d-1}^{2} \in O\left(c^{d^{d+1}}\right),
$$

where $c \approx 1.26408$ (see Lemma 5.5).

We can explicitly state a sharper conjecture, that was checked by the computer classification of Kreuzer and Skarke [19], [20] for four-dimensional reflexive polytopes:

Conjecture 1.7. Let $d \geq 4$. The reflexive simplex $S_{Q_{d}^{\prime}}$ has solely the largest number of lattice points among all d-dimensional lattice polytopes with only one interior lattice point.

Our second main result is another indication in this direction. It has been observed by Haase and Melnikov [12] for $d \leq 4$ and initialized this research:

Theorem B. The maximal number of lattice points on an edge of a reflexive simplex of dimension d is $2 t_{d-1}+1$, with equality attained only for $S_{Q_{d}^{\prime}}$.

Here is the third main result (its first part can be seen as a variant of the BlaschkeSantaló inequality for centered compact convex bodies, see p. 165 of [23]):

Theorem C. Let $P$ be a d-dimensional reflexive simplex. Then

$$
(d+1)^{d+1} \leq \operatorname{Vol}(P) \operatorname{Vol}\left(P^{*}\right) \leq t_{d}^{2},
$$


with equality of the first bound iff the sum of the vertices of $P$ equals 0 , and equality of the second bound iff $P \cong S_{Q_{d}}$ (in this case $P \cong P^{*}$ ). Furthermore, let the vertices of $P^{*}$ generate the lattice $N$. Then

$$
\operatorname{Vol}\left(P^{*}\right) \leq t_{d}
$$

with equality iff $P^{*} \cong S_{Q_{d}} \cong P$.

The proofs of Theorems A, B, and C are given in the last section.

\section{The Main Results, Algebro-Geometric}

In this section we reformulate Theorems $\mathrm{A}, \mathrm{B}$, and $\mathrm{C}$ algebro-geometrically.

For $Q \in \mathbb{N}_{>0}^{d+1}$ we denote by $\mathbb{P}(Q)$ the associated weighted projective space (see p. 35 of [9]) with weights $Q$, e.g., $\mathbb{P}(1, \ldots, 1)=\mathbb{P}^{d}$. For any $d \geq 2$ the weighted projective spaces $\mathbb{P}\left(Q_{d}\right)$ and $\mathbb{P}\left(Q_{d}^{\prime}\right)$, where $Q_{d}$ and $Q_{d}^{\prime}$ were defined in Definition 1.3, have Gorenstein singularities, i.e., the anticanonical divisor is an ample Cartier divisor. This follows for instance from Theorem 4.3 below.

Here is our first main algebro-geometric result:

Theorem $\mathbf{A}^{\prime}$. Let $X$ be ad-dimensional $\mathbb{Q}$-factorial Gorenstein toric Fano variety with Picard number one.

1. If $d=2$, then

$$
\left(-K_{X}\right)^{2} \leq 9
$$

with equality iff $X \cong \mathbb{P}^{2}$.

2. If $d=3$, then

$$
\left(-K_{X}\right)^{3} \leq 72
$$

with equality iff $X \cong \mathbb{P}(3,1,1,1)$ or $X \cong \mathbb{P}\left(Q_{3}^{\prime}\right)=\mathbb{P}(6,4,1,1)$.

3. If $d \geq 4$, then

$$
\left(-K_{X}\right)^{d} \leq 2 t_{d-1}^{2}
$$

with equality iff $X \cong \mathbb{P}\left(Q_{d}^{\prime}\right)$.

Since Gorenstein toric Fano varieties have canonical singularities, this result motivates the following more general conjecture:

Conjecture 2.1. The results of Theorem $A^{\prime}$ hold for Gorenstein Fano varieties with canonical singularities.

In the case of threefolds the bound in Theorem $\mathrm{A}^{\prime}$ is the so-called Fano-Iskovskikh conjecture. It has very recently been proven by Prokhorov [26]. 
Recall that the class number of a $d$-dimensional toric variety $X$, i.e., the rank of the group of Weil divisors modulo linear equivalence, is equal to the number of onedimensional cones of the associated fan minus $d$ (see p. 63 of [9]). $X$ is $\mathbb{Q}$-factorial, i.e., the Picard number equals the class number, if and only if all cones of the associated fan are simplices. Now, a theorem of Batyrev [2, Theorem 4.1.9] yields that $X$ is a $d$ dimensional Gorenstein toric Fano variety if and only if the the associated fan $\triangle$ (in $N$ ) is spanned by the faces of a $d$-dimensional reflexive polytope. In this case, $X$ is $\mathbb{Q}$-factorial with Picard number one, or, equivalently, $X$ has class number one, if and only if $\Delta$ is spanned by the faces of a reflexive simplex. Then we denote its dual reflexive simplex by $P$. Here, the vertices of the simplex $P^{*}$ generate the lattice $N$ if and only if $X$ is isomorphic to a weighted projective space (e.g., see Theorem 3.3).

Definition 2.2. If $X=\mathbb{P}(Q)$ (for $Q \in \mathbb{N}_{>0}^{d+1}$ ) is a weighted projective space with Gorenstein singularities, then we define $S_{Q}$ as the (up to unimodular equivalence unique) reflexive simplex $P \subseteq M_{\mathbb{R}}$.

This definition is consistent with Definition 1.4 due to Theorem 4.3 and Proposition 4.4 .

Now Theorem A' follows directly from Theorem A due to the following formula (see p. 111 of [9]):

$$
\left(-K_{X}\right)^{d}=\operatorname{Vol}(P) .
$$

The second algebro-geometric theorem is concerned with the maximal anticanonical degree of integral torus-invariant curves (in contrast, in Mori theory one usually is rather interested in their minimal degree, see Proposition 2.1 of [22]) :

Theorem B'. Let $X$ be a d-dimensional $\mathbb{Q}$-factorial Gorenstein toric Fano variety with Picard number one. Let $C$ be a torus-invariant integral curve on $X$. Then

$$
-K_{X} \cdot C \leq 2 t_{d-1},
$$

where equality implies $X \cong \mathbb{P}\left(Q_{d}^{\prime}\right)$.

To see how to obtain this result from Theorem B, we associate to $X$, as before, a fan $\triangle$ in $N$ and a reflexive simplex $P \subseteq M_{\mathbb{R}}$. We remark that any torus-invariant integral curve $C$ is given by a wall $\rho$, i.e., a $(d-2)$-dimensional cone of $\triangle$. Moreover, $\rho$ is obviously in correspondence with an edge $e$ of $P$. Hence, Theorem $\mathrm{B}^{\prime}$ follows from the following observation (see Corollary 3.6 of [22]):

$$
-K_{X} \cdot C=|e \cap M|-1 .
$$

For the last main algebro-geometric result we need the following definition:

Definition 2.3. If $P \subseteq M_{\mathbb{R}}$ is a reflexive polytope and $X$ is the Gorenstein toric Fano variety associated to the fan (in $N$ ) spanned by the faces of $P^{*}$, we define $X^{*}$ as the Gorenstein toric Fano variety that is associated to the fan (in $M$ ) spanned by the faces of $P$. 
Theorem $\mathbf{C}^{\prime}$. Let $X$ be a d-dimensional $\mathbb{Q}$-factorial Gorenstein toric Fano variety with Picard number one. Then

$$
(d+1)^{d+1} \leq\left(-K_{X}\right)^{d}\left(-K_{X^{*}}\right)^{d} \leq t_{d}^{2},
$$

where equality of the lower bound is attained only if $X$ is a quotient of $\mathbb{P}^{d}$ by a finite abelian group, and equality of the upper bound is attained only for $X \cong \mathbb{P}\left(Q_{d}\right)$ (in this case $X \cong X^{*}$ ).

Furthermore, let $X$ be a weighted projective space with Gorenstein singularities. Then

$$
\left(-K_{X^{*}}\right)^{d} \leq t_{d},
$$

with equality iff $X \cong \mathbb{P}\left(Q_{d}\right) \cong X^{*}$.

One could conjecture that the first part of this result, as well as Theorem $\mathrm{B}^{\prime}$, might also be true for arbitrary Gorenstein toric Fano varieties.

Theorem $\mathrm{C}^{\prime}$ is a direct translation of Theorem C (use (1), and the basic results of the next section, notably Theorem 3.2 and (3)).

\section{Lattice Simplices and Weight Systems}

The next two sections summarize and extend results of Batyrev [2, Sections 5.4 and 5.5] and Conrads [5].

Throughout let $P \subseteq M_{\mathbb{R}}$ be a $d$-dimensional rational simplex containing the origin of the lattice in the interior. We define $\mathcal{V}(P)$ as the set of vertices of $P$ and $\mathcal{F}(P)$ as the set of facets of $P$. For $F \in \mathcal{F}(P)$ we let $\eta_{F} \in N_{\mathbb{R}}$ denote the unique inner normal of $F$ with $\left\langle\eta_{F}, F\right\rangle=-1$, thus, $\eta_{F} \in \mathcal{V}\left(P^{*}\right)$. In general, $\operatorname{conv}(V)$ denotes the convex hull of a set $V \subseteq M_{\mathbb{R}}$.

\section{Definition 3.1.}

- Let $P=\operatorname{conv}\left(v_{0}, \ldots, v_{d}\right)$. We define $q_{i}:=\left|\operatorname{det}\left(v_{j}: j=0, \ldots, d, j \neq i\right)\right|$ for $i=0, \ldots, d$. The family $Q_{P}:=\left(q_{0}, \ldots, q_{d}\right)$ is called the associated weight system of $P$ of total weight $\left|Q_{P}\right|:=q_{0}+\cdots+q_{d}$. If $P$ is a lattice polytope, we define the factor of $P$ as the index of the sublattice of $M$ generated by the vertices $v_{0}, \ldots, v_{d}$.

- We explicitly define a weight system $Q$ of length $d$ as a $(d+1)$-tuple of positive rational numbers; two weight systems are regarded as isomorphic, if they are equal up to permutation. The total weight $|Q|$ is the sum of the elements of $Q$.

Let $Q$ consist of natural numbers. Then we define the factor of $Q$ as $\lambda_{Q}:=$ $\operatorname{gcd}(Q)$. In this case $Q$ is called reduced, if $\lambda_{Q}=1$. We denote by $Q_{\text {red }}$ its reduction $Q / \lambda_{Q} . \quad Q$ is called normalized if $\operatorname{gcd}(Q \backslash\{i\})=1$ for $i=0, \ldots, d$. 
If $P$ is a lattice simplex, we have, by Lemma 2.4 of [5],

$$
\lambda_{P}=\lambda_{Q_{P}} \in \mathbb{N}_{>0} .
$$

Furthermore, in this case $\left(Q_{P}\right)_{\text {red }}=\left(q_{0}^{\prime}, \ldots, q_{n}^{\prime}\right)$ is the unique reduced weight system satisfying

$$
\sum_{i=0}^{d} q_{i}^{\prime} v_{i}=0
$$

Now here is the following theorem (see 4.4-4.7 of [5] and Theorem 5.4.5 of [2]):

Theorem 3.2 (Batyrev, Conrads). For a reduced weight system $Q$, there exists, up to isomorphism, a unique lattice simplex $P_{Q} \subseteq M_{\mathbb{R}}$ with $Q_{P_{Q}}=Q$. The toric variety associated to the fan spanned by the faces of $P_{Q}$ is isomorphic to $\mathbb{P}(Q)$.

Furthermore, if $P$ is a lattice simplex with $\left(Q_{P}\right)_{\mathrm{red}}=Q$, then there exists (in a lattice basis) a matrix $H$ in Hermite normal form with determinant $\lambda_{P}$ such that $P \cong H P_{Q}$. In particular, any complete toric variety with class number one is the quotient of a weighted projective space by the action of a finite abelian group.

Regarding normalized weight systems gives uniqueness (see Corollaries 3.7 and 3.8 of [5]):

Theorem 3.3 (Conrads). There is a correspondence between isomorphism classes of

- lattice simplices $P$ that contain the origin and whose vertices are primitive lattice points that generate the lattice,

- normalized weight systems $Q$, and

- weighted projective spaces,

that is given by $P \mapsto Q_{P}$ and $Q \mapsto \mathbb{P}(Q)$.

There is now an important invariant of a weight system, this generalizes Definition 5.4 of [5] as can be seen from Proposition 4.5(2):

Definition 3.4. Let $Q$ be a weight system of length $d$. Then we define

$$
m_{Q}:=\frac{|Q|^{d-1}}{q_{0} \cdots q_{d}} \in \mathbb{Q}_{>0} .
$$

For the next result we need a straightforward lemma: 


\section{Lemma 3.5.}

$$
\begin{gathered}
\operatorname{det}\left(\begin{array}{ccccccc}
\left(n_{1}-1\right) & -1 & \cdots & & & \cdots & -1 \\
& \ddots & \ddots & \ddots & & & \\
-1 & \cdots & -1 & \left(n_{i}-1\right) & -1 & \cdots & -1 \\
& & & \ddots & \ddots & \ddots & \\
-1 & \cdots & & & \cdots & -1 & \left(n_{d}-1\right)
\end{array}\right) \\
=n_{1} \cdots n_{d}-\sum_{j=1}^{d} \prod_{i=1, i \neq j}^{d} n_{i} . \\
\end{gathered}
$$

Dualizing changes the associated weight system only by a factor:

\section{Proposition 3.6.}

$$
Q_{P^{*}}=m_{Q_{P}} Q_{P}
$$

Proof. Let $Q:=Q_{P}, t:=|Q|$, and $\mathcal{V}(P)=\left\{v_{0}, \ldots, v_{d}\right\}$. For $i=0, \ldots, d$ we denote by $F_{i}=\operatorname{conv}\left(v_{j}: j=0, \ldots, d, j \neq i\right)$ the facet of $P$ not containing $v_{i}$. Fix a $\mathbb{Z}$-basis of $M$ and its dual basis of $N$. Now let $i \in\{0, \ldots, d\}$, and let $A_{i}$ be the matrix consisting of the coordinates of $v_{j}(j=0, \ldots, d, j \neq i)$ as rows, and let $B_{i}$ be the matrix consisting of the coordinates of $\eta_{F_{j}}(j=0, \ldots, d, j \neq i)$ as columns. Since $v_{i}=-\sum_{j \neq i}\left(q_{j} / q_{i}\right) v_{j}$, we get

$$
\left\langle\eta_{F_{i}}, v_{i}\right\rangle=\sum_{j \neq i} \frac{q_{j}}{q_{i}}=\frac{t-q_{i}}{q_{i}}=\frac{t}{q_{i}}-1 .
$$

Without restriction we set $i=0$. Applying the previous lemma to $A_{0} B_{0}$, we get

$$
\operatorname{det}\left(A_{0} B_{0}\right)=\frac{t}{q_{1}} \cdots \frac{t}{q_{d}}\left(1-\sum_{j=1}^{d} \frac{q_{j}}{t}\right)=\frac{t}{q_{1}} \cdots \frac{t}{q_{d}}\left(1-\frac{t-q_{0}}{t}\right)=\frac{t^{d-1}}{q_{1} \cdots q_{d}} q_{0} .
$$

Therefore $\operatorname{det}\left(B_{0}\right)=\operatorname{det}\left(A_{0} B_{0}\right) / \operatorname{det}\left(A_{0}\right)=t^{d-1} /\left(q_{1} \cdots q_{d}\right)=m_{Q} q_{0}$.

\section{Reflexive Simplices and Unit Partitions}

We consider the case of a reflexive simplex. Here, the following definitions turn out to be convenient (the notion of unit partitions is closely related to the much-studied subject of Egyptian fractions, where the denominators have to be pairwise different, see Section D11 of [11]):

\section{Definition 4.1.}

- A weight system is called reflexive if it is reduced and any weight is a divisor of the total weight. Especially it has to be normalized.

- A family of positive natural numbers $\left(k_{0}, \ldots, k_{d}\right)$ is called a unit partition of total weight $t^{\prime}:=\operatorname{lcm}\left(k_{0}, \ldots, k_{d}\right)$ if $\sum_{i=0}^{d} 1 / k_{i}=1$. 
Now there is the following observation (essentially due to Batyrev [2, Section 5.4]), that is straightforward to prove:

Proposition 4.2. In the notation of the previous definition we have that mapping $\left(q_{0}, \ldots, q_{d}\right)$ to $\left(t / q_{0}, \ldots, t / q_{d}\right)$, respectively mapping $\left(k_{0}, \ldots, k_{d}\right)$ to $\left(t^{\prime} / k_{0}, \ldots, t^{\prime} / k_{d}\right)$, yields a bijection between reflexive weight systems and unit partitions.

The notion of a reflexive weight system is motivated by the following result $[5$, Proposition 5.1] (partially [2, Theorem 5.4.3]):

Theorem 4.3 (Batyrev, Conrads). Under the correspondence of Theorem 3.3 we get correspondences of isomorphism classes of

- reflexive simplices whose vertices generate the lattice,

- reflexive weight systems, respectively, unit partitions, and

- weighted projective spaces with Gorenstein singularities.

Recall that if $Q$ is a reflexive weight system, then the reflexive simplex $S_{Q}$, as defined in Definition 2.2, is isomorphic to $\left(P_{Q}\right)^{*}$. It is difficult to give in general an explicit description of $S_{Q}$, see [5]. However, in the case in which the vertices of some facet of $P_{Q}$ form a lattice basis, we can conveniently describe $P_{Q}$ and $S_{Q}$, see Definition 1.4 (the proof is left to the reader):

Proposition 4.4. Let $Q$ be a reflexive weight system with weight $q_{d}=1$. Denote the corresponding unit partition by $\left(k_{0}, \ldots, k_{d}\right)$. Then we have

$$
\begin{gathered}
P_{Q} \cong \operatorname{conv}\left(e_{0}, \ldots, e_{d-1},-q_{0} e_{0}-\cdots-q_{d-1} e_{d-1}\right), \\
S_{Q} \cong\left(P_{Q}\right)^{*} \cong \operatorname{conv}\left(k_{0} e_{0}-u, \ldots, k_{d-1} e_{d-1}-u,-u\right),
\end{gathered}
$$

for $u:=e_{0}+\cdots+e_{d-1}$, where $e_{0}, \ldots, e_{d-1}$ is a $\mathbb{Z}$-basis of $M$.

We give the following generalization of Lemma 5.3 and Propostion 5.5 of [5]:

Proposition 4.5. Let $P \subseteq M_{\mathbb{R}}$ be a reflexive simplex with associated weight system $Q:=Q_{P}=\lambda_{P} Q_{\text {red. }}$. Then $Q_{\text {red }}$ is a reflexive weight system. Let $P_{\mathrm{red}} \subseteq M_{\mathbb{R}}$ be the corresponding reflexive simplex and let $\left(k_{0}, \ldots, k_{d}\right)$ be the associated unit partition. We have the following results:

1. $\left(Q_{P^{*}}\right)_{\mathrm{red}}=Q_{\mathrm{red}}, \lambda_{P^{*}}=m_{Q_{P}} \lambda_{P}=m_{Q_{\mathrm{red}}} / \lambda_{P}$.

$$
m_{Q_{\mathrm{red}}}=\frac{k_{0} \cdots k_{d}}{\operatorname{lcm}\left(k_{0}, \ldots, k_{d}\right)^{2}}=\lambda_{\left(P_{\mathrm{red}}\right)^{*}} \in \mathbb{N}_{>0} .
$$

3. There exist injective lattice homomorphisms inducing bijections

$$
P_{\mathrm{red}} \stackrel{\lambda_{P}}{\rightarrow} P \stackrel{m_{Q_{\mathrm{red}}} / \lambda_{P}}{\longrightarrow}\left(P_{\mathrm{red}}\right)^{*} \cong S_{Q_{\mathrm{red}}},
$$


where the number on the arrow gives the integer determinant of the respective lattice homomorphism.

4. $\lambda_{P} \mid m_{Q_{\text {red }}}$. Furthermore,

$$
\lambda_{P}=1 \Longleftrightarrow P \cong P_{\text {red }}, \quad \lambda_{P}=m_{Q_{\text {red }}} \Longleftrightarrow P \cong\left(P_{\text {red }}\right)^{*} .
$$

5.

$$
\operatorname{Vol}(P)=\left|Q_{P}\right| \leq \operatorname{Vol}\left(S_{Q_{\text {red }}}\right)=\frac{k_{0} \cdots k_{d}}{\operatorname{lcm}\left(k_{0}, \ldots, k_{d}\right)},
$$

with equality iff $P \cong S_{Q_{\mathrm{red}}}$.

Proof. We can easily see that $Q_{\text {red }}$ is a reflexive weight system (or use Proposition 5.1 of [5]). Result 1 follows from Proposition 3.6, (3), and Definition 3.4. Result 2 follows from the construction of $\left(k_{0}, \ldots, k_{d}\right)$, Definition 3.4, and result 1. Result 3: First apply Theorem 3.2 to $P$. Then apply Theorem 3.2 to the lattice simplex $P^{*}$, use result 1 and dualize. Result 4 follows from result 3. Result 5: The (normalized) volume of a $d$ dimensional simplex having the origin as a vertex is the absolute value of the determinant of the matrix formed by the vertices different from the origin. Hence $\operatorname{Vol}(P)=\left|Q_{P}\right|=$ $\lambda_{P}\left|Q_{\text {red }}\right|=\lambda_{P} \operatorname{lcm}\left(k_{0}, \ldots, k_{d}\right)$. Now use results 2 and 3 .

The second point of the proposition was already proven by Batyrev [2, Corollary 5.5.4] when investigating fundamental groups.

Corresponding to the special reflexive weight systems defined in Definition 1.3 we have some important unit partitions:

\section{Definition 4.6.}

- $\left(y_{0}, \ldots, y_{d-1}, t_{d}\right)$ is called the Sylvester partition of length $d$. It is a unit partition of total weight $t_{d}=t_{d-1} y_{d-1}$ corresponding to the reflexive weight system $Q_{d}$. It corresponds to a self-dual reflexive simplex, since $m_{Q_{d}}=1$.

- $\left(y_{0}, \ldots, y_{d-2}, 2 t_{d-1}, 2 t_{d-1}\right)$ is called the enlarged Sylvester partition of length $d$. It corresponds to $Q_{d}^{\prime}$, and we have $m_{Q_{d}^{\prime}}=t_{d-1}$.

As an illustration of the previous notions we classify all five isomorphism classes of two-dimensional reflexive simplices:

Example 4.7. Let $d=2$. We have three unit partitions:

1. $(3,3,3)$ corresponding to $Q:=(1,1,1)$. Here, $m_{Q}=3$. So we have $P_{Q} \cong$ $\operatorname{conv}\left((1,0),(0,1),(-1,-1)\right.$ (corresponding to $\left.\mathbb{P}^{2}\right)$ and $S_{Q} \cong\left(P_{Q}\right)^{*} \cong$ $\operatorname{conv}((2,-1),(-1,2),(-1,-1))$ as the only reflexive simplices $P$ with $\left(Q_{P}\right)_{\text {red }}=$ $Q$ (due to Proposition 4.5(4)).

2. The Sylvester partition $(2,3,6)$ corresponding to $Q:=Q_{2}=(3,2,1)$. Here, $m_{Q}=1$. So this yields the self-dual reflexive simplex $P_{Q} \cong S_{Q} \cong$ $\operatorname{conv}((1,0),(0,1),(-3,-2))$ (corresponding to $\mathbb{P}(Q))$. 
3. The enlarged Sylvester partition $(2,4,4)$ corresponding to $Q:=Q_{2}^{\prime}=(2,1,1)$. Here, $m_{Q}=2$. So we have $P_{Q} \cong \operatorname{conv}((1,0),(0,1),(-2,-1)$ (corresponding to $\mathbb{P}(Q))$ and $S_{Q} \cong\left(P_{Q}\right)^{*} \cong \operatorname{conv}((1,-1),(-1,3),(-1,-1))$ as the only reflexive simplices $P$ with $\left(Q_{P}\right)_{\text {red }}=Q$.

\section{The Main Results, Number-Theoretic}

In this section we prove the following result on unit partitions:

Theorem 5.1. Let $\left(k_{0}, \ldots, k_{d}\right)$ be a unit partition.

1.

$$
\operatorname{lcm}\left(k_{0}, \ldots, k_{d}\right)^{2} \leq k_{0} \cdots k_{d} \leq t_{d}^{2},
$$

2.

with equality in the second case only for the Sylvester partition.

$$
(d+1)^{d+1} \leq k_{0} \cdots k_{d},
$$

with equality iff $\left(k_{0}, \ldots, k_{d}\right)=(d+1, \ldots, d+1)$.

3. Let $d \geq 3$ and $k_{0} \leq \cdots \leq k_{d}$. Then

$$
\frac{k_{0} \cdots k_{d}}{\operatorname{lcm}\left(k_{0}, \ldots, k_{d}\right)} \leq k_{0} \cdots k_{d-1} \leq 2 t_{d-1}^{2}
$$

with equality in the second case iff $\left(k_{0}, \ldots, k_{d}\right)$ is the enlarged Sylvester partition or $(2,6,6,6)$.

First we recall the most important classical result about unit partitions due to Curtiss [6, Theorem I]:

Theorem 5.2 (Curtiss). Let $a_{1}, \ldots, a_{m}$ be positive integers such that $s:=\sum_{i=1}^{m}\left(1 / a_{i}\right)<$ 1. Then

$$
s \leq \sum_{i=0}^{m-1} \frac{1}{y_{i}}=1-\frac{1}{t_{m}},
$$

with equality iff $\left\{a_{1}, \ldots, a_{m}\right\}=\left\{y_{0}, \ldots, y_{m-1}\right\}$.

As a corollary we get that $\max \left(k_{0}, \ldots, k_{d}\right) \leq t_{d}$ for any unit partition $\left(k_{0}, \ldots, k_{d}\right)$, and equality holds only for the Sylvester partition.

While the proof of Curtiss was quite complicated, the following nice result [16, Lemma 1] has a much simpler proof, and it easily yields again the previous theorem. Here we have included some statements that are implicit in their proof. 
Lemma 5.3 (Izhboldin, Kurliandchik). Let $x_{1}, \ldots, x_{n}$ be real numbers satisfying $x_{1} \geq$ $x_{2} \geq \cdots \geq x_{n} \geq 0, x_{1}+\cdots+x_{n}=1$, and $x_{1} \cdots x_{k} \leq x_{k+1}+\cdots+x_{n}$ fork $=1, \ldots, n-1$. Then

$$
x_{n} \geq \frac{1}{t_{n-1}}, \quad x_{1} \cdots x_{n} \geq \frac{1}{t_{n-1}^{2}},
$$

where equality in the first case holds iff equality in the second case holds iff $x_{i}=1 / y_{i-1}$ for $i=1, \ldots, n-1$.

Any unit partition fulfills (after reordering) the assumptions of the lemma: To see this let $\left(k_{0}, \ldots, k_{d}\right)$ be a unit partition with $k_{0} \leq \cdots \leq k_{d}$. We define $x_{i}:=1 / k_{i}$ for $i=0, \ldots, d$. Then for $i=0, \ldots, d-1$ we get

$$
\begin{aligned}
0 & <x_{i+1}+\cdots+x_{d}=1-x_{0}-\cdots-x_{i}=\frac{k_{0} \cdots k_{i}-\sum_{j=0}^{i} \prod_{l=0, l \neq j}^{i} k_{l}}{k_{0} \cdots k_{i}} \\
& \geq \frac{1}{k_{0} \cdots k_{i}}=x_{0} \cdots x_{i} .
\end{aligned}
$$

Hence, the previous lemma proves the upper bound (and its equality case) in statement 1 of Theorem 5.1. The middle inequality in statement 1 follows from Proposition 4.5(2). Statement 2 of Theorem 5.1 follows immediately from applying the inequality of arithmetic and geometric means to $1 / k_{0}, \ldots, 1 / k_{d}$.

For the proof of the last point in Theorem 5.1 we need a preliminary result:

Lemma 5.4. Let $n \geq 4$, and $1 \leq r \leq n-1$. Then

$$
(r+1)^{r} t_{n-r-1}^{r+1} \leq 2 t_{n-2}^{2},
$$

with equality iff $r=1$ or $(n, r)=(4,2)$.

Proof. Proof by induction on $n$. By explicitly checking $n=4,5$, we can assume $n \geq 6$.

For $r=1$ the statement is trivial, so let $r \geq 2$. By induction hypothesis for $(n-1, r-1)$ we have $r^{r-1} t_{n-r-1}^{r} \leq 2 t_{n-3}^{2}$, this yields $(r+1)^{r} t_{n-r-1}^{r+1} \leq 2 t_{n-3}^{2} t_{n-r-1}((r+1) / r)^{r} r$. Since $((r+1) / r)^{r}<e$, it is enough to show $t_{n-3}^{2} t_{n-r-1} e r \leq t_{n-2}^{2}$, or, equivalently, $t_{n-r-1}$ er $\leq y_{n-3}^{2}$.

For $n \geq 6$ it is easy to see that $e(n-1) \leq y_{n-3}$ (e.g., by Lemma 5.5 below). Hence, $t_{n-r-1} e r \leq t_{n-3} e(n-1) \leq t_{n-3} y_{n-3}<y_{n-3}^{2}$.

The following lemma gives the doubly exponential behavior of the Sylvester sequence (e.g., $[10,(4.17)])$ :

Lemma 5.5. There is a constant $c \approx 1.2640847353 \cdots$ (called the Vardi constant, see A076393 of [28]) such that for any $n \in \mathbb{N}$,

$$
y_{n}=\left\lfloor c^{2^{n+1}}+1 / 2\right\rfloor .
$$

Now using Lemma 5.4 and the ideas of the proof of Lemma 5.3 we can show the following result, which immediately implies statement 3 in Theorem 5.1: 
Lemma 5.6. Let $n \geq 4$, and let $x:=\left(x_{1}, \ldots, x_{n}\right)$ as in Lemma 5.3. Then

$$
x_{1} \cdots x_{n-1} \geq \frac{1}{2 t_{n-2}^{2}},
$$

with equality iff either $n=4$ and $x_{1}=\frac{1}{2}, x_{2}=x_{3}=x_{4}=\frac{1}{6}$, or $\left(1 / x_{1}, \ldots, 1 / x_{n}\right)$ equals the enlarged Sylvester partition of length $n-1$.

Proof. The proof splits into three parts.

Part I

Let $A$ denote the set of $n$-tuples $x$ satisfying the conditions of Lemma 5.3. It is easy to see that we have for $x \in A$ necessarily $1>x_{1}$ and $x_{n}>0$.

Since $A$ is compact, there exists some $x \in A$ with $x_{1} \cdots x_{n-1}$ minimal. Because of $\left(1 / y_{0}, \ldots, 1 / y_{n-3}, 1 /\left(2 t_{n-2}\right), 1 /\left(2 t_{n-2}\right)\right) \in A$ we have $x_{1} \cdots x_{n-1} \leq 1 /\left(2 t_{n-2}^{2}\right)$.

We show that $x_{n-1}=x_{n}$.

So assume $x_{n-1}>x_{n}$.

Claim. In this case we have

$$
\begin{gathered}
x_{1}>x_{2}>\cdots>x_{n-1}>x_{n}, \\
x_{1} \cdots x_{k}=x_{k+1}+\cdots+x_{n} \quad \text { for } \quad k=1, \ldots, n-2 .
\end{gathered}
$$

Proof of the Claim. By convention we set $x_{0}:=1$ and $x_{n+1}:=0$. We proceed by induction on $l=1, \ldots, n-1$, and assume by induction hypothesis that $x_{1}>x_{2}>$ $\cdots>x_{l} \geq x_{l+1}$ and $x_{1} \cdots x_{k}=x_{k+1}+\cdots+x_{n}$ for $k=1, \ldots, l-2$.

We distinguish three cases:

1. $x_{l}>x_{l+1}$ and $x_{1} \cdots x_{l-1}=x_{l}+\cdots+x_{n}$.

In this case we can proceed.

2. $x_{l}>x_{l+1}$ and $x_{1} \cdots x_{l-1}<x_{l}+\cdots+x_{n}$.

This implies $l \geq 2$. Then we can find some $\delta>0$ s.t. $x^{\prime} \in A$ with $x_{l-1}^{\prime}:=x_{l-1}+\delta$, $x_{l}^{\prime}:=x_{l}-\delta$ and $x_{j}^{\prime}:=x_{j}$ for $j \in\{1, \ldots, n\} \backslash\{l-1, l\}$. Hence, $x_{1}^{\prime} \cdots x_{n-1}^{\prime}=$ $\left(\left(x_{1} \cdots x_{n-1}\right) /\left(x_{l-1} x_{l}\right)\right)\left(x_{l-1} x_{l}+\delta\left(x_{l}-x_{l-1}\right)-\delta^{2}\right)<x_{1} \cdots x_{n-1}$, a contradiction.

3. $x_{l}=x_{l+1}=\cdots=x_{i}>x_{i+1}$ for $l+1 \leq i \leq n-1$.

This implies $l \leq n-2$. Again we find some $\delta>0$ s.t. $x^{\prime} \in A$ with $x_{l}^{\prime}:=x_{l}+\delta$, $x_{i}^{\prime}:=x_{i}-\delta$, and $x_{j}^{\prime}:=x_{j}$ for $j \in\{1, \ldots, n\} \backslash\{l, i\}$.

This can be done, since otherwise there has to exist $l \leq j<i$ such that $x_{1} \cdots x_{j}=x_{j+1}+x_{j+2}+\cdots+x_{n}$. Since $x_{j}=x_{j+1}$ we have $0=\left(1-x_{1} \cdots x_{j-1}\right) x_{j}+$ $x_{j+2}+\cdots+x_{n}$, a contradiction.

Since again $x_{1}^{\prime} \cdots x_{n-1}^{\prime}<x_{1} \cdots x_{n-1}$, we get a contradiction.

So we have $x_{1}=x_{2}+\cdots+x_{n}=1-x_{1}$, hence $x_{1}=1 / 2=1 / y_{0}$. In the same way we have for $k=2, \ldots, n-2$ that $x_{1} \cdots x_{k}=x_{k+1}+\cdots+x_{n}=1-x_{1}-\cdots-x_{k}$, hence we get by induction $\left(1 / t_{k-1}\right) x_{k}=1-1 / y_{0}-\cdots-1 / y_{k-2}-x_{k}=1 / t_{k-1}-x_{k}$, thus $x_{k}=1-t_{k-1} x_{k}$, this implies $x_{k}=1 /\left(1+t_{k-1}\right)=1 / y_{k-1}$. So this yields $x_{1}=$ $1 / y_{0}, \ldots, x_{n-2}=1 / y_{n-3}$. 
Furthermore, $x_{n-1}+x_{n}=1-x_{1}-\cdots-x_{n-2}=1 / t_{n-2}$. Since $x_{n-1}>x_{n}$, we get $x_{n-1}>1 /\left(2 t_{n-2}\right)$. Therefore we have proven $x_{1} \cdots x_{n-1}>1 /\left(2 t_{n-2}^{2}\right)$, a contradiction.

Hence, $x_{n-1}=x_{n}$.

\section{Part II}

Let $A^{\prime}$ denote the set of $(n-1)$-tuples $w \in \mathbb{R}^{n-1}$ satisfying the following conditions: $w_{1} \geq w_{2} \geq \cdots \geq w_{n-2} \geq w_{n-1} / 2 \geq 0, w_{1}+\cdots+w_{n-1}=1$ and $w_{1} \cdots w_{k} \leq$ $w_{k+1}+\cdots+w_{n-1}$ for $k=1, \ldots, n-2$.

Let $w \in A^{\prime}$ be fixed with $w_{1} \cdots w_{n-1}$ minimal.

Since $\left(1 / y_{0}, \ldots, 1 / y_{n-3}, 1 / t_{n-2}\right) \in A^{\prime}$ due to $n \geq 4$, we have $w_{1} \cdots w_{n-1} \leq 1 / t_{n-2}^{2}$.

We show $w=\left(\frac{1}{2}, \frac{1}{6}, \frac{1}{3}\right)$ or $w=\left(1 / y_{0}, \ldots, 1 / y_{n-3}, 1 / t_{n-2}\right)$; in particular, $w_{1} \cdots w_{n-1}=1 / t_{n-2}^{2}$.

Let $z:=w_{s}=\cdots=w_{n-2}=w_{n-1} / 2$ for $1 \leq s \leq n-1$ minimal.

We define $r:=n-s$. There are three cases to consider:

1. $s=1$, i.e., $r=n-1$.

Then $1=w_{1}+\cdots+w_{n-2}+w_{n-1}=(n-2) z+2 z=n z$, so $z=1 / n$. This implies $w_{1} \cdots w_{n-1}=2 / n^{n-1}$. However, $1 / t_{n-2}^{2}<2 / n^{n-1}$ for $n \geq 4$ by Lemma 5.4, a contradiction.

2. $s=2$, i.e., $r=n-2$.

Then $1=w_{1}+w_{2}+\cdots+w_{n-2}+w_{n-1}=w_{1}+(n-3) z+2 z=w_{1}+(n-1) z$, hence $w_{1}=1-(n-1) z$. Since $w_{1}>z$, we get $z<1 / n$. On the other hand, $w_{1} \leq w_{2}+\cdots+w_{n-1}=(n-1) z$, hence $z \geq 1 /(2(n-1))$.

We have $w_{1} \cdots w_{n-1}=(1-(n-1) z) 2 z^{n-2}$. This function attains its minimum on the interval $[1 /(2(n-1)), 1 / n$ [ only for $z=1 /(2(n-1))$. (The elementary analytical proof of this statement is left to the reader.)

Therefore $1 / t_{n-2}^{2} \geq w_{1} \cdots w_{n-1} \geq 1 /(2(n-1))^{n-2}$. However, from Lemma 5.4 we get $1 /(2(n-1))^{n-2} \geq 1 / t_{n-2}^{2}$, with equality only for $n=4$ and $r=2$. Hence this yields $z=1 /(2(n-1))$ and $w=\left(\frac{1}{2}, \frac{1}{6}, \frac{1}{3}\right)$.

3. $s \geq 3$, i.e., $r \leq n-3$.

Now a similar reasoning as in the proof of the claim in Part I yields

$$
w_{1}=\frac{1}{y_{0}}, \ldots, w_{s-2}=\frac{1}{y_{s-3}} .
$$

Then $1=w_{1}+\cdots+w_{s-2}+w_{s-1}+w_{s}+\cdots+w_{n-1}=1-1 / t_{s-2}+w_{s-1}+$ $(n-s+1) z$, hence $w_{s-1}=1 / t_{s-2}-(r+1) z$.

Since $w_{s-1}>z$, we get $z<1 /\left((r+2) t_{s-2}\right)$.

Since $w_{1} \cdots w_{s-2} w_{s-1} \leq w_{s}+\cdots+w_{n-1}$, we get $\left(1 / t_{s-2}\right)\left(1 / t_{s-2}-(r+1) z\right) \leq$ $(r+1) z$, hence

$$
z \geq \frac{1}{(r+1) t_{s-2}^{2}\left(1+\frac{1}{t_{s-2}}\right)}=\frac{1}{(r+1) t_{s-1}}
$$

Now we define $f(z):=\left(1 / t_{s-2}\right)\left(1 / t_{s-2}-(r+1) z\right) 2 z^{r}=w_{1} \cdots w_{n-1}$. 
Since the function $f(z)$ is for $z>0$ strictly monotone increasing up to some value and then strictly monotone decreasing, we see that

$$
\begin{aligned}
\frac{1}{t_{n-2}^{2}} & \geq w_{1} \cdots w_{n-1} \geq \min \left(f\left(\frac{1}{(r+1) t_{s-1}}\right), f\left(\frac{1}{(r+2) t_{s-2}}\right)\right) \\
& =\min \left(\frac{2}{(r+1)^{r} t_{s-1}^{r+1}}, \frac{2}{(r+2)^{r+1} t_{s-2}^{r+2}}\right) .
\end{aligned}
$$

There are two cases:

(a) $s=n-1$, i.e., $r=1$.

Here, $w_{1} \cdots w_{n-1} \geq \min \left(1 / t_{n-2}^{2}, 2 /\left(9 t_{n-3}^{3}\right)\right)$. Due to $n \geq 4$ and $t_{n-2}=$ $t_{n-3}\left(t_{n-3}+1\right)$ we have $1 / t_{n-2}^{2} \leq 2 /\left(9 t_{n-3}^{3}\right)$, thus $w_{1} \cdots w_{n-1}=1 / t_{n-2}^{2}$, and $z=$ $1 /\left(2 t_{n-2}\right)$, since $z<1 /\left((r+2) t_{s-2}\right)$. Hence $w=\left(1 / y_{0}, \ldots, 1 / y_{n-3}, 1 / t_{n-2}\right)$. (b) $s \leq n-2$, i.e., $r \geq 2$.

Here, $2 /\left((r+1)^{r} t_{s-1}^{r+1}\right) \leq 2 /\left((r+2)^{r+1} t_{s-2}^{r+2}\right)$ if and only if $y_{s-2}^{r+1} / t_{s-2} \geq$ $(r+2)^{r+1} /(r+1)^{r}$. This is true for $r=2$. For $r \geq 3$ we have

$$
(r+2)\left(\frac{r+2}{r+1}\right)^{r}<(r+2) e<3^{r} \leq y_{s-2}^{r}<\frac{y_{s-2}^{r+1}}{t_{s-2}} .
$$

Hence $1 / t_{n-2}^{2} \geq w_{1} \cdots w_{n-1} \geq 2 /\left((r+1)^{r} t_{s-1}^{r+1}\right)$, a contradiction to Lemma 5.4.

\section{Part III}

We finish the proof.

Let $n \geq 4$ and $x=\left(x_{1}, \ldots, x_{n}\right)$ satisfy the conditions in Lemma 5.3, where $x_{1} \cdots x_{n-1}$ has minimal value. By Part I we have $x_{n-1}=x_{n}$. Hence, $w^{\prime}:=\left(x_{1}, \ldots, x_{n-2}, 2 x_{n-1}\right)$ is an element in $A^{\prime}$, so by Part II we get

$$
x_{1} \cdots x_{n-1}=\frac{w_{1}^{\prime} \cdots w_{n-1}^{\prime}}{2} \geq \frac{1}{2 t_{n-2}^{2}},
$$

where equality yields $\left(x_{1}, \ldots, x_{n}\right)=\left(1 / y_{0}, \ldots, 1 / y_{n-3}, 1 /\left(2 t_{n-2}\right), 1 /\left(2 t_{n-2}\right)\right)$ or $n=4$ and $\left(x_{1}, x_{2}, x_{3}, x_{4}\right)=\left(\frac{1}{2}, \frac{1}{6}, \frac{1}{6}, \frac{1}{6}\right)$.

\section{Proofs of Theorems A, B, and C}

Combining the results of the previous two sections we get:

\section{Corollary 6.1.}

1. If $Q$ is a reflexive weight system of length $d$, then

$$
|Q| \leq t_{d}, \quad \text { with equality iff } Q \text { is isomorphic to } Q_{d} .
$$

2. If $P$ is a reflexive simplex, then

$$
(d+1)^{d+1} \leq\left|Q_{P}\right|\left|Q_{P^{*}}\right|=\operatorname{Vol}(P) \operatorname{Vol}\left(P^{*}\right) \leq t_{d}^{2},
$$

with equality of the first bound iff the sum of the vertices of $P$ equals zero, and equality of the second bound iff $P \cong S_{Q_{d}}\left(\cong S_{Q_{d}}^{*}\right.$ ). 
3. If $P$ is a reflexive simplex for $d \geq 3$, then

$$
\left|Q_{P}\right|=\operatorname{Vol}(P) \leq 2 t_{d-1}^{2},
$$

with equality iff $P \cong S_{Q_{\text {red }}}$ for $Q_{\text {red }}$ isomorphic to $Q_{d}^{\prime}$ or $(3,1,1,1)$.

Proof. 1. Follows from Theorem 5.1(1), since $|Q|=\operatorname{lcm}\left(k_{0}, \ldots, k_{d}\right)$ for the corresponding unit partition.

2. We observe that for $Q_{P}=\lambda_{P} Q_{\text {red }}$ Proposition 4.5(1),(2) yields $Q_{P^{*}}=$ $\left(m_{Q_{\text {red }}} / \lambda_{P}\right) Q_{\text {red }}$ and $\left|Q_{P} \| Q_{P^{*}}\right|=k_{0} \cdots k_{d}$, where $\left(k_{0}, \ldots, k_{d}\right)$ is the unit partition corresponding to $Q_{\text {red }}$. Now the statements follow from Proposition 4.5(5), Theorem 5.1(1,2), equation (3), and the fact that $P_{Q_{d}} \cong S_{Q_{d}}$ is the only reflexive simplex $P$ with $\left(Q_{P}\right)_{\text {red }}=Q_{d}$, since $m_{Q_{d}}=1$.

3. Follows from Proposition 4.5(5) and Theorem 5.1(3).

Eventually, we can finish the proofs of the three main theorems:

Proof of Theorem A. The case $d=2$ was considered in Example 4.7. For $d \geq 3$ use Corollary 6.1(3). Furthermore, note that for $d=3$ we have the well-known formula $|P \cap M|=\operatorname{Vol}(P) / 2+3$, that can be derived for instance from [14].

In the proof of Theorem B we use the following observation (see Section 2.2 of [8]):

Proposition 6.2. Let $Q$ be a normalized weight system with associated $\operatorname{simplex} P_{Q}=$ $\operatorname{conv}\left(v_{0}, \ldots, v_{d}\right)$. Let $i, j \in\{0, \ldots, d\}, i \neq j$. Then we have for the torus-invariant integral curve $C$ on $\mathbb{P}(Q)$ associated to the wall $\operatorname{pos}\left(v_{k}: i \neq k \neq j\right)$ :

$$
\left(-K_{\mathbb{P}(Q)}\right) \cdot C=\frac{|Q|}{\operatorname{lcm}\left(q_{i}, q_{j}\right)} .
$$

In particular, when $Q$ is a reflexive weight system with corresponding unit partition $\left(k_{0}, \ldots, k_{d}\right)$, we get

$$
\left(-K_{\mathbb{P}(Q)}\right) \cdot C=\operatorname{gcd}\left(k_{i}, k_{j}\right)
$$

Proof of Theorem $B$. The fact that $S_{Q_{d}^{\prime}}$ satisfies the bound is trivial from the definition, see Proposition 4.4.

Let $P=\operatorname{conv}\left(v_{0}, \ldots, v_{d}\right) \subseteq M_{\mathbb{R}}$ be a reflexive simplex with associated weight system $Q_{P}$, and let $Q:=\left(Q_{P}\right)_{\text {red }}=\left(q_{0}, \ldots, q_{d}\right)$ correspond to the unit partition $\left(k_{0}, \ldots, k_{d}\right)$. Let $v_{d}, v_{d-1}$ be the vertices of an edge $e$ of $P$ that contains the maximal number $|e \cap M|$. Thus, $c:=|e \cap M|-1=\operatorname{gcd}\left(k_{d}, k_{d-1}\right)$ by formula (2) and Proposition 6.2. We may assume $k_{d} \geq k_{d-1}$ and $c \geq 2 t_{d-1}$.

Now Theorem 5.2 implies $1-1 / k_{d-1}-1 / k_{d}=\sum_{i=0}^{d-2} 1 / k_{i} \leq 1-1 / t_{d-1}$, so $2 / k_{d-1} \geq 1 / k_{d-1}+1 / k_{d} \geq 1 / t_{d-1}$. This implies $2 t_{d-1} \leq c \leq k_{d-1} \leq 2 t_{d-1}$, so we have $c=2 t_{d-1}=k_{d-1}=k_{d}$. In particular, we get $\sum_{i=0}^{d-2} 1 / k_{i}=1-1 / t_{d-1}$, thus, Theorem 5.2 yields $\left\{k_{0}, \ldots, k_{d-2}\right\}=\left\{y_{0}, \ldots, y_{d-2}\right\}$. Hence $Q=Q_{d}^{\prime}$.

By Proposition 4.4 we can choose $\mathcal{V}\left(P_{\text {red }}\right)=\left\{e_{0}, \ldots, e_{d-1}, e_{d}:=-q_{0} e_{0}-\cdots-\right.$ $\left.q_{d-1} e_{d-1}\right\}$ for some $\mathbb{Z}$-basis $e_{0}, \ldots, e_{d-1}$ of $M$. By Theorem 3.2 there is (up to unimodular 
equivalence) a $d \times d$ matrix $H=\left\{h_{i, j}\right\}$ in Hermite normal form of determinant $\lambda_{P}$ such that $H\left(e_{0}\right)=v_{0}, \ldots, H\left(e_{d-1}\right)=v_{d-1}, H\left(e_{d}\right)=v_{d}$. Recall that a quadratic matrix $H$ is in Hermite normal form, if it is a lower triangular matrix with natural numbers as coefficients such that $h_{i, j}<h_{j, j}$ for $i>j$.

Since $v_{d-1}$ is primitive, we get $h_{d, d}=1$, so $v_{d-1}=e_{d-1}$. Because of

$$
\frac{1}{2 t_{d-1}}\left(H\left(e_{d}\right)-e_{d-1}\right) \in M \quad \text { and } \quad \frac{q_{i}}{2 t_{d-1}}=\frac{1}{y_{i}} \quad \text { for } \quad i=0, \ldots, d-2
$$

we get the following $d-1$ equations:

$$
\begin{aligned}
& \frac{h_{1,1}}{y_{0}} \in \mathbb{N}, \\
& y_{0}\left(\frac{h_{2,1}}{y_{0}}+\frac{h_{2,2}}{y_{1}}\right) \in \mathbb{N}, \\
& \cdots\left.\cdots+\frac{h_{d-1, d-1}}{y_{d-2}}\right) \\
& y_{0} \cdots y_{d-3}\left(\frac{h_{d-1,1}}{y_{0}}+\cdots\right.
\end{aligned}
$$

Using the fact that $\operatorname{gcd}\left(y_{i}, y_{j}\right)=1$ for $i \neq j$, we deduce by induction that $y_{i-1}$ divides $h_{i, i}$ for $i=1, \ldots, d-1$. Hence, we have

$$
m_{Q}=t_{d-1}=y_{0} \cdots y_{d-2} \leq h_{1,1} \cdots h_{d-1, d-1} h_{d, d}=\operatorname{det} H=\lambda_{P} \leq m_{Q} .
$$

Now Proposition 4.5(4) implies $P \cong\left(P_{\text {red }}\right)^{*} \cong S_{Q_{d}^{\prime}}$.

Proof of Theorem $C$. The first statement follows from Corollary 6.1(2), and the second statement from Corollary 6.1(1) and $\operatorname{Vol}(P)=\left|Q_{P}\right|$, since in this case $Q_{P}$ is already reduced, thus a reflexive weight system. Also note $P_{Q_{d}} \cong S_{Q_{d}}$.

\section{Acknowledgements}

The author thanks his thesis advisor Victor Batyrev for giving reference to [26], C. Haase for his interest, D. Ploog for discussion, and A. Hermann for helpful conversation, as well as the anonymous referees and G. Ziegler for suggestions on the organization of the paper.

Most of this work is part of the author's thesis.

\section{References}

1. V.V. Batyrev, Boundedness of the degree of multidimensional toric Fano varieties, Moscow Univ. Math. Bull. 37 (1982), 28-33.

2. V.V. Batyrev, Dual polyhedra and mirror symmetry for Calabi-Yau hypersurfaces in toric varieties, J. Algebraic Geom. 3 (1994), 493-535.

3. H. Blichfeldt, A new principle in the geometry of numbers, with some applications, Trans. Amer. Math. Soc. 15 (1914), 227-235.

4. A.A. Borisov and L.A. Borisov, Singular toric Fano varieties, Math. USSR-Sb. 75 (1993), 277-283. 
5. H. Conrads, Weighted projective spaces and reflexive polytopes, Manuscripta Math. 107 (2002), 215-227.

6. D.R. Curtiss, On Kellogg's diophantine problem, Amer. Math. Monthly 29 (1922), 380-387.

7. O. Debarre, Fano varieties, in K. Böröczky, J. Kollár, and T. Szamuely, eds., Higher Dimensional Varieties and Rational Points, Bolyai Society Mathematical Studies 12, Springer-Verlag, Berlin, 2003, pp. 93-132.

8. O. Fujino, Notes on toric varieties from Mori theoretic viewpoint, Tohoku Math. J. 55 (2003), 551-564.

9. W. Fulton, Introduction to Toric Varieties, Annals of Mathematics Studies 131, Princeton University Press, Princeton, NJ, 1993.

10. R.L. Graham, D.E. Knuth, and O. Patashnik, Concrete Mathematics: A Foundation for Computer Science, Addison-Wesley, Reading, MA, 1989.

11. R.K. Guy, Unsolved Problems in Number Theory, Problem Books in Mathematics, Unsolved Problems in Intuitive Mathematics, Vol. 1, Springer-Verlag, New York, 1981.

12. C. Haase and I.V. Melnikov, The reflexive dimension of a lattice polytope, Preprint, math.CO/0406485, 2004.

13. D. Hensley, Lattice vertex polytopes with interior lattice points, Pacific J. Math. 105 (1983), 183-191.

14. T. Hibi, Dual polytopes of rational convex polytopes, Combinatorica 12 (1992), 237-240.

15. J.-M. Hwang, On the degrees of Fano four-folds of Picard number 1, J. Reine Angew. Math. 556 (2003), 225-235.

16. O. Izhboldin and L. Kurliandchik, Unit fractions, AMS Transl. Ser. 2166 (1995), 193-200.

17. A.M. Kasprzyk, Toric Fano three-folds with terminal singularities, Tohoku Math. J. 58 (2006), 101-121.

18. M. Kreuzer and H. Skarke, Complete classification of reflexive polyhedra in four dimensions, Adv. Theor. Math. Phys. 4 (2000), 1209-1230.

19. M. Kreuzer and H. Skarke, PALP: A package for analyzing lattice polytopes with applications to toric geometry, Comput. Phys. Commun. 157 (2004), 87-106.

20. M. Kreuzer and H. Skarke, Calabi-Yau data, http://hep.itp.tuwien.ac.at/ kreuzer/CY.

21. J.C. Lagarias and G.M. Ziegler, Bounds for lattice polytopes containing a fixed number of interior points in a sublattice, Canad. J. Math. $\mathbf{4 3}$ (1991), 1022-1035.

22. R. Laterveer, Linear systems on toric varieties, Tohoku Math. J. 48 (1996), 451-458.

23. E. Lutwak, Selected affine isoperimetric inequalities, in P.M. Gruber and J.M. Wills, eds., Handbook of Convex Geometry, Volume A, North-Holland, Amsterdam, 1993, pp. 151-176.

24. B. Nill, Gorenstein toric Fano varieties, Manuscripta Math. 116 (2005), 183-210.

25. O. Pikhurko, Lattice points inside lattice polytopes, Mathematika 48 (2001), 15-24.

26. Y.G. Prokhorov, The degree of Fano threefolds with canonical Gorenstein singularities, Sb. Math. 196 (2005), 77-114.

27. B. Reznick, Clean lattice tetrahedra, Preprint, math.CO/0606227, 2006.

28. N.J.A. Sloane, On-line encyclopedia of integer sequences, http://www.research.att.com/ njas/sequences/.

29. J. Zaks, M. Perles, and J. Wills, On lattice polytopes having interior lattice points, Elem. Math. 37 (1982), $44-46$.

Received April 6, 2006, and in revised form August 11, 2006. Online publication February 12, 2007. 